OPEN

SUBJECT AREAS:

VIRAL EPIDEMIOLOGY

VIRAL EVOLUTION

Received

10 January 2014

Accepted

13 February 2014

Published

3 March 2014

Correspondence and requests for materials should be addressed to

Z.R.D. (ynepidzr@ sohu.com)

* These authors contributed equally to this work.

\section{Complete Genome Characterization of a Novel Enterovirus Type EV-B 106 Isolated in China, 2012}

Jingjing Tang ${ }^{*}$, Zexin Tao ${ }^{2 *}$, Zhengrong Ding', Yong Zhang ${ }^{3}$, Jie Zhang' ', Bingiun Tian', Zhixian Zhao', Lifen Zhang' \& Wenbo $\mathrm{Xu}^{3}$

\footnotetext{
'Yunnan Center for Disease Control and Prevention, Kunming, Yunnan Province, People's Republic of China, ${ }^{2}$ Shandong Provincial Key Laboratory of Infectious Disease Control and Prevention, Shandong Center for Disease Control and Prevention, Jinan, Shandong Province, People's Republic of China, ${ }^{3}$ WHO WPRO Regional Polio Reference Laboratory and Ministry of Health Key Laboratory for Medical Virology, National Institute for Viral Disease Control and Prevention, Chinese Center for Disease Control and Prevention, Beijing, People's Republic of China.
}

Human enterovirus B106 (EV-B106) is a recently identified member of enterovirus species B. In this study, we report the complete genomic characterization of an EV-B106 strain (148/YN/CHN/12) isolated from an acute flaccid paralysis patient in Yunnan Province, China. The new strain had 79.2-81.3\% nucleotide and 89.1-94.8\% amino acid similarity in the VP1 region with the other two EV-B106 strains from Bolivia and Pakistan. When compared with other EV serotypes, it had the highest (73.3\%) VP1 nucleotide similarity with the EV-B77 prototype strain CF496-99. However, when aligned with all EV-B106 and EV-B77 sequences available from the GenBank database, two major frame shifts were observed in the VP1 coding region, which resulted in substantial (20.5\%) VP1 amino acid divergence between the two serotypes.

Phylogenetic analysis and similarity plot analysis revealed multiple recombination events in the genome of this strain. This is the first report of the complete genome of EV-B106.

he genus Enterovirus in the family Picornaviridae is a group of nonenveloped positive-sense RNA viruses that cause a wide range of diseases in humans and other mammals. Most human enterovirus (EV) infections are asymptomatic or result in only mild diseases such as the common cold or minor undifferentiated febrile illnesses; yet under certain conditions, EVs also cause serious human diseases such as acute flaccid paralysis (AFP); meningitis; encephalitis; myocarditis; and hand, foot, and mouth disease $\mathrm{e}^{1-4}$.

The EV genome is about $7.5 \mathrm{~kb}$ in length. It has a single open reading frame (ORF) flanked by $5^{\prime}$ and $3^{\prime}$ untranslated regions (UTRs). The 5' UTR is about 740-nucleotides (nt) long and contains an internal ribosomebinding site, which is essential for translation initiation ${ }^{5-7}$. The $3^{\prime}$ UTR, approximately 100 -nt long, forms highly conserved secondary and tertiary structures that are important for initiation of replication ${ }^{8-10}$. The ORF is translated into a polyprotein of 2200 amino acids (aa), which is processed by viral proteases into structural (VP4, VP2, VP3, and VP1) and non-structural (2A, 2B, 2C, 3A, 3B, 3C, and 3D) proteins ${ }^{11}$. Current EV classification is based on the molecular typing method which suggests strains with $<70 \%$ VP1 nt similarity are classified as different types and the strains with $>75 \%$ VP1 nt similarity are classified as members of same type. This method has been shown to correspond with serotype neutralization ${ }^{12,13}$. For the nt similarities in 'grey zone' of $70-75 \%$, Brown et al. suggested that a more stringent value of $88 \%$ VP1 amino acid identity is more appropriate for routine typing ${ }^{14}$.

The genus Enterovirus consists of 12 species, 7 of which (EV-A to D, rhinovirus - A to C) are associated with human infections. EV-B consists of 61 types $^{11,15}$. Molecular typing of serologically untypable strains has led to the discovery of a large number of new EV types ${ }^{16-19}$. Enterovirus B106 (EV-B106) is a newly identified member of EV-B. To date, only two partial sequences of EV-B106, from Bolivia and Pakistan, have been available in the GenBank database ${ }^{20}$.

Yunnan Province is located in southwest China, with an area of 390,000 square kilometers and a population of 45,966 million (2010 census data). It borders Vietnam, Laos, and Myanmar. In a previous study, we reported the identification and molecular epidemiology of new EV types isolated in Yunnan Province, including EV-A76, EVB75, EV-B80, EV-B81, EV-B83, EV-B93, and EV-C96 ${ }^{21}$. Here, we report the identification and genomic char- 


\begin{tabular}{|c|c|c|c|c|c|}
\hline Region & PAK NIH SP1 202 & EV-B & EV-A & EV-C & EV-D \\
\hline $5^{\prime}$ UTR & N/A & $79.7-89.1$ & $68.8-79.0$ & $54.8-69.3$ & $63.5-65.1$ \\
\hline VP4 & 78.4 & $67.9-83.2$ & $55.3-61.2$ & $57.8-66.1$ & $56.8-60.2$ \\
\hline VP2 & 82.4 & $64.7-74.6$ & $47.7-52.2$ & $54.7-58.7$ & $50.9-54.5$ \\
\hline VP3 & 79.7 & $61.6-76.8$ & $46.9-51.0$ & $53.9-58.2$ & $47.9-48.8$ \\
\hline VP1 & 81.3 & $54.9-73.1$ & $39.3-43.3$ & $44.3-49.7$ & $44.6-45.6$ \\
\hline $2 \mathrm{~A}$ & N/A & $75.5-81.7$ & $55.9-61.3$ & $54.4-59.1$ & $51.6-53.1$ \\
\hline $2 B$ & N/A & $74.7-83.5$ & $53.5-60.6$ & $49.4-57.5$ & $58.5-60.9$ \\
\hline $2 C$ & N/A & $79.6-84.1$ & $60.8-64.3$ & $57.5-62.6$ & $63.6-64.4$ \\
\hline $3 \mathrm{~A}$ & N/A & $73.4-82.7$ & $52.8-59.5$ & $54.3-60.6$ & $59.5-62.1$ \\
\hline $3 B$ & N/A & 69.6-83.3 & $53.0-63.3$ & $54.5-72.7$ & $65.0-65.2$ \\
\hline $3 C$ & N/A & $75.0-84.5$ & $56.1-59.5$ & $59.3-63.7$ & $59.7-63.9$ \\
\hline $3 \mathrm{D}$ & N/A & $79.2-84.7$ & $61.8-64.1$ & $64.8-68.9$ & $65.8-66.0$ \\
\hline 3'UTR & N/A & $77.7-87.1$ & $23.0-31.4$ & $22.8-45.7$ & $31.7-36.4$ \\
\hline
\end{tabular}

acterization of an EV-B106 strain (148/YN/CHN/12; hereafter referred to as strain $12148 / \mathrm{YN}$ ) recovered from one patient with AFP in Yunnan Province, China, in 2012.

\section{Results}

Isolation, molecular typing, and VP1 sequence analysis. Strain 12148/YN was isolated on both RD and HEp-2 cells. VP1 region sequencing and BLAST analysis indicated that the type of this strain is EV-B106. The VP1-coding sequence of this strain showed 81.3\% nt and $94.8 \%$ aa similarity with that of the EV-B106 Pakistan strain PAK_NIH_SP_1202. Only a 303-nt partial VP1 sequence is available for another EV-B106 strain, BOL/03-10665A from Bolivia; nevertheless, homologous comparison based on this region revealed that the Yunnan strain had $79.2 \%$ nt and $89.1 \%$ aa similarity with BOL/03-10665A. Compared with the VP1 sequences of the prototype strains of other EV-B types, strain $12148 / \mathrm{YN}$ had the highest similarity to the EV-B77 prototype strain CF496-99 (AY493062), with $73.3 \% \mathrm{nt}$ and $79.5 \%$ aa identity.

Whole genome analysis. The whole genome length of strain 12148/ YN was 7,420 nt. A large ORF (6,570 nt), encoding a potential polyprotein precursor of 2,192 aa, was flanked by $5^{\prime}$ and $3^{\prime}$ UTRs of $742 \mathrm{nt}$ and $99 \mathrm{nt}$, respectively. The overall base compositions of the genomes were $27.5 \% \mathrm{~A}, 25.2 \% \mathrm{G}, 23.5 \% \mathrm{C}$, and $23.9 \% \mathrm{U}$. Table 1 shows the nucleotide sequence identities of different regions of the genome between strain 12148/YN and the EV-B106 Pakistan strain

(a)

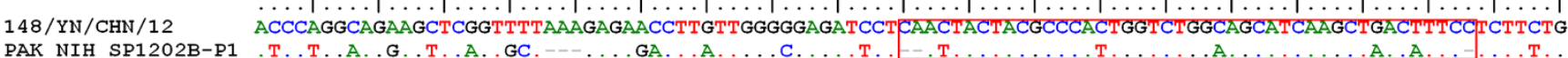

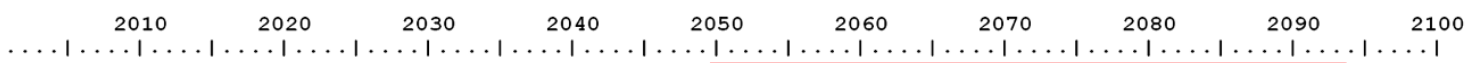

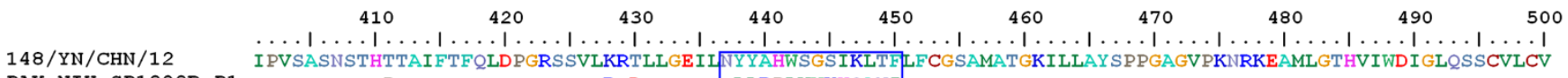

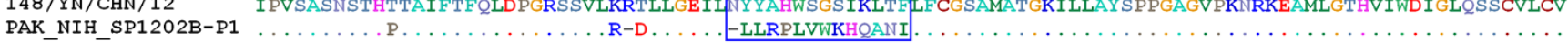

(b)

EV77 CF496-99 AJ493062 EV77 CAF-MAM-03-048 JN255662 EV77 KCD85 JX513592 EV77 AY208119 EV77 USA-TX97-10394 AY843302 EV106 PAK NIH SP1202B-P1 $148 / \mathrm{YN} / \mathrm{CHN} / 12$ EV77 NIV1023591LV404 JX476192 G..A. . . . T.G......G

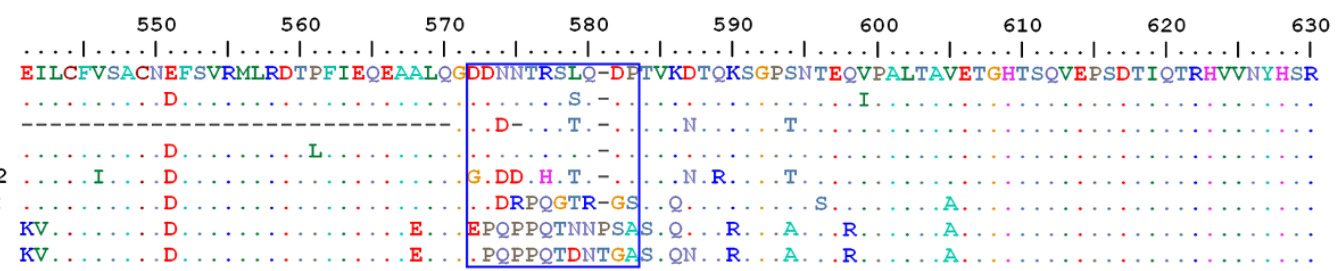

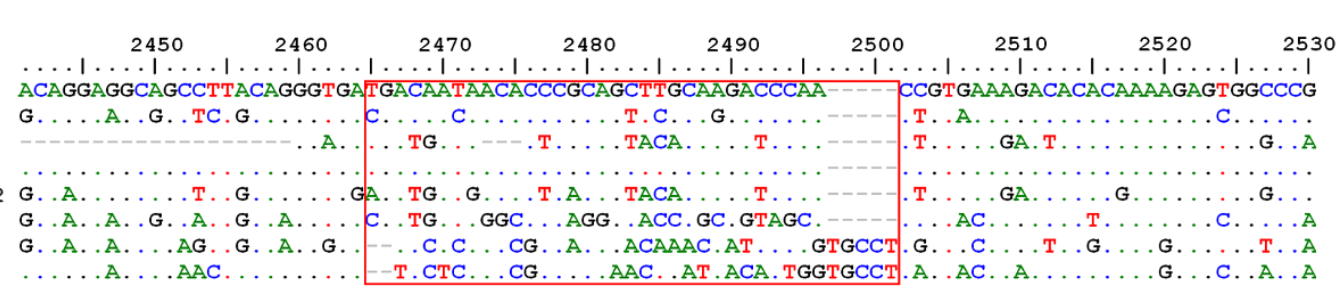

EV77 CF496-99 AJ493062 EV77 CAF-MAM-03-048 JN255662 EV77 KCD85 JX513592 EV77 AY208119 EV77 USA-TX97-10394 AY843302 EV106 PAK NIH SP1202B-P1 $148 / \mathrm{YN} / \mathrm{CHN} / 12$ EV77 NIV1023591LV404 JX476192

Figure $1 \mid$ Nucleotide insertions and deletions and the resulted amino acid changes in the P1 region among EV-B106 strains (a) and with EV-B77

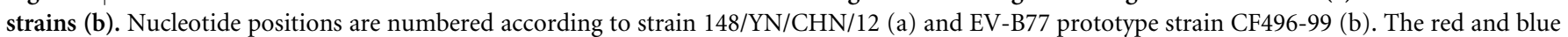
squares indicate the nucleotide frame shifts and associated amino acid changes, respectively. 
(only the $P 1$ sequence available) and prototype strains of the species EV-A, EV-B, EV-C, and EV-D. The entire list of prototype strains used in the analysis can be found as Supplementary Table S1. Strain 12148 /YN had high similarity in the VP1 coding region with the Pakistan strain, confirming that the two strains belong to the same type ( $>75 \%$ VP1 nt sequence identity within a type). In contrast, it shared $54.9-73.1 \%$ nt sequence identity in the VP1 coding region with the other EV-B types, and less than $50 \%$ with the other species (EV-A, EV-C, EV-D).

Following alignment with the $P 1$ coding region of the EV-B106 strain PAK_NIH_SP_1202, three insertions were observed: a 3-nt "AAA" insertion at positions 2,023-2,025 (numbering according to the whole genome of strain $12148 / \mathrm{YN}$ ), a 2 -nt " $\mathrm{CA}$ " insertion at positions 2,050-2,051, and a 1-nt "C" insertion at position 2,093 (Fig. 1). All the insertions are located in the VP3 coding region. Because EV-B77 has the highest sequence homology with EVB106, alignment among the EV-B106 strains 12148/YN, PAK_NIH_ SP_1202, and all EV-B77 strains available in the GenBank database was conducted, and frame shifts between the two types were found (Fig. 1), including two major frame shifts observed in the VP1 coding region, which result in substantial (up to 20.5\%) VP1 aa divergence between the two types.

Phylogenetic and recombination analysis with EV-B prototype strains. Phylogenetic analysis based on the $P 1, P 2$, and $P 3$ coding regions of strain $12148 / \mathrm{YN}$, the Pakistan strain $(P 1$ region sequence only), and all EV-B prototype strains available in the GenBank database was conducted (Fig. 2). As expected, strain 12148/YN formed a lineage with the Pakistan strain in the tree for the P1 coding region, with a bootstrap value of $100 \%$ (Fig. 2a), which confirmed the preliminary molecular typing results. Meanwhile, the two EV-B106 strains clustered together with the closely related strain, EV-B77, having a 100\% bootstrap value. However, strain 12148 /YN did not cluster with any of the other EV types in the trees for the $P 2$ and $P 3$ coding regions (with high bootstrap support) (Fig. $2 \mathrm{~b}$ and $2 \mathrm{c}$ ), which reflected that one or more putative recombination events between EV-B106 and other EV-B types might have occurred.

To confirm the existence of recombination events involving strain $12148 / \mathrm{YN}$, the similarity plot and bootscanning analysis were conducted with the other EV-B prototype strains (Fig. 3). The results revealed recombination between strain 12148/YN and the EV-B strains at the $P 2$ and $P 3$ coding regions. One recombination event between $12148 / \mathrm{YN}$ and EV-B107 was observed in the $2 B$ region (about nt positions 3,700-3,960).

Recombination analysis with closely related strains. Recombination analysis was also performed for strain $12148 / \mathrm{YN}$ with other EV$B$ strains. Because of the large number of EV-B genome sequences available in the GenBank database, closely related sequences were screened using BLAST online. Since recombination events usually occur in non-structural coding regions ${ }^{22,23}$, the $5^{\prime} \mathrm{UTR}, P 2$, and $P 3$ regions of strain $12148 / \mathrm{YN}$ were separately analyzed using BLAST, and the sequences with the highest similarities were used in the recombination analysis. The sequences used in the study were derived from the following: E4 strain AUS250G (FJ172447) from Australia $^{24}$; the E6 strain KM57/09 (JQ801739) from Yunnan Province, China; the E9 strain DM33 (KC238667) from the (a)

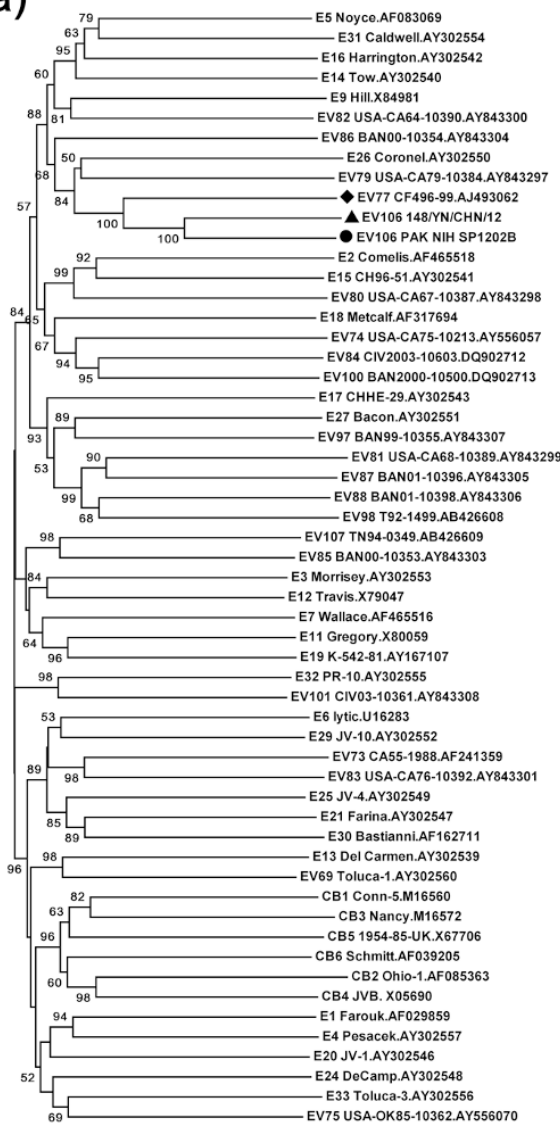

$\longmapsto 0.05$ (b)

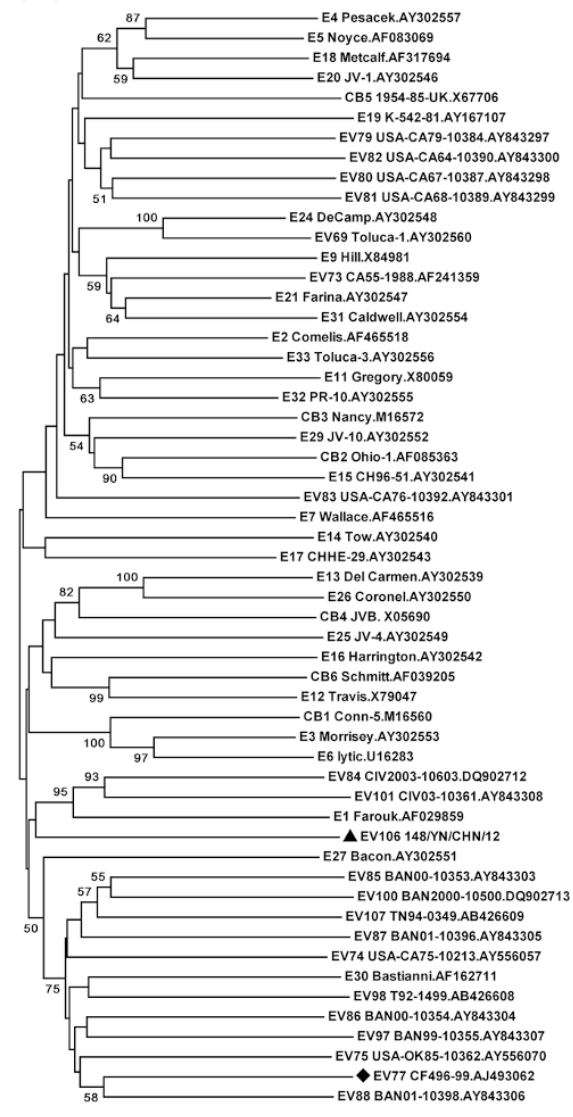

$\widehat{0.02}$ (c)

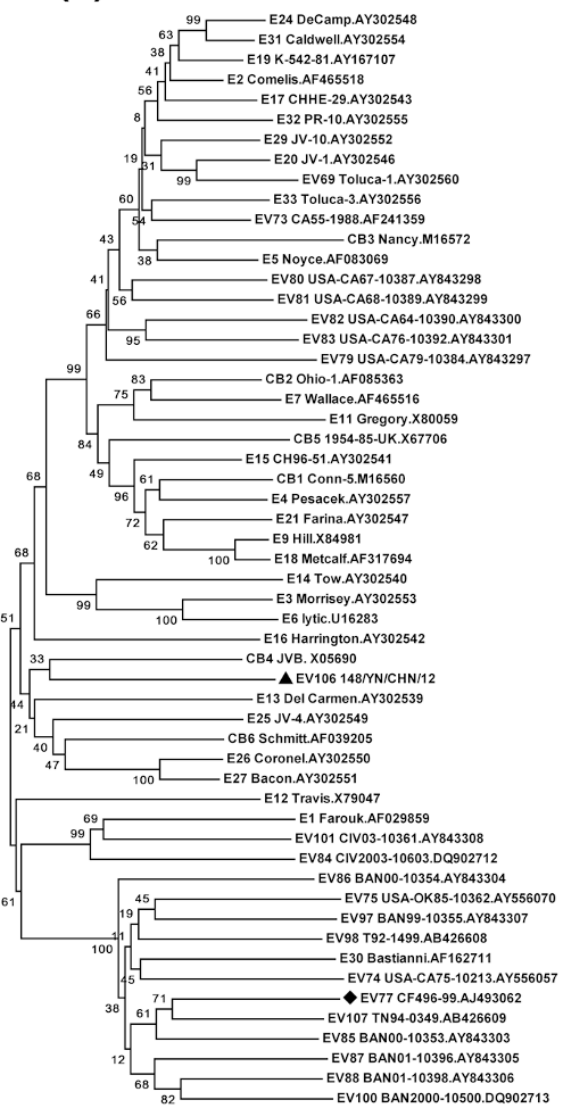

$\longdiv { 0 . 0 2 }$

Figure $2 \mid$ Phylogenetic relationships of the EV-B106 strains and other EV-B prototype strains. The phylogenetic trees based on nucleotide sequences for $P 1$ (a), P2 (b), and P3 (c) coding regions were constructed from the nucleotide sequence alignment using the neighbor-joining algorithm of the MEGA 4.0 software. 


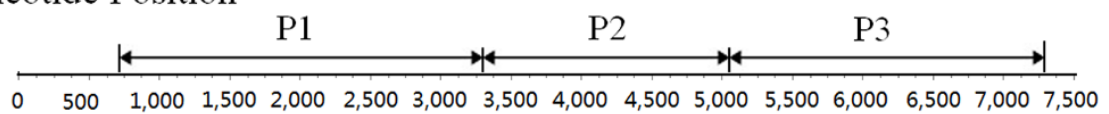

(a)

a)

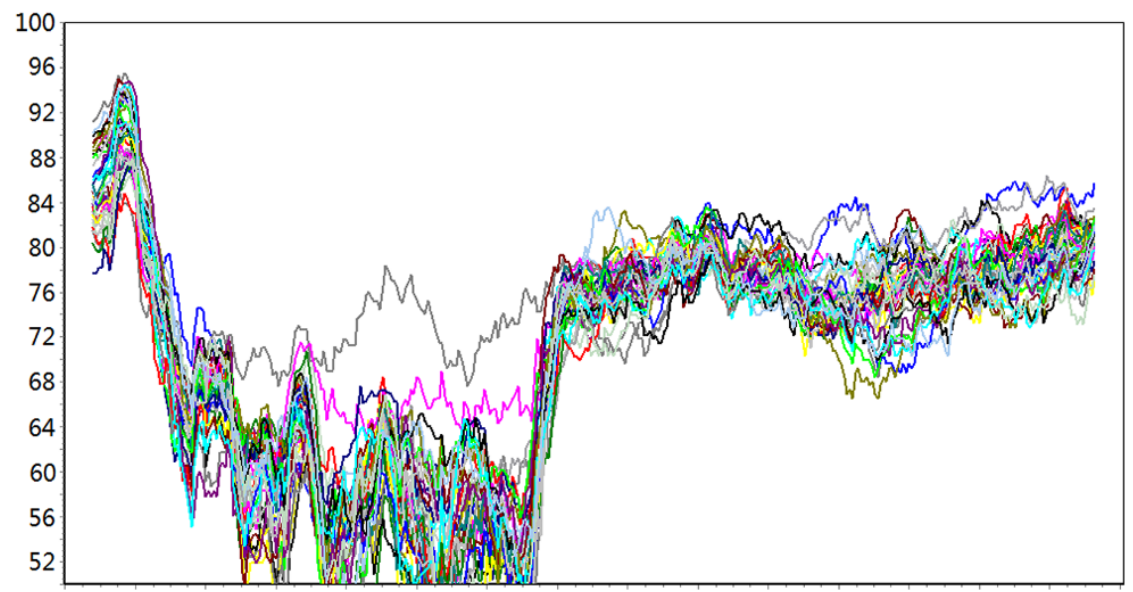

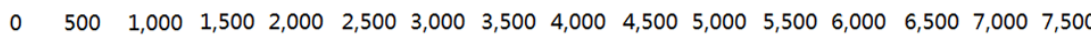

Position
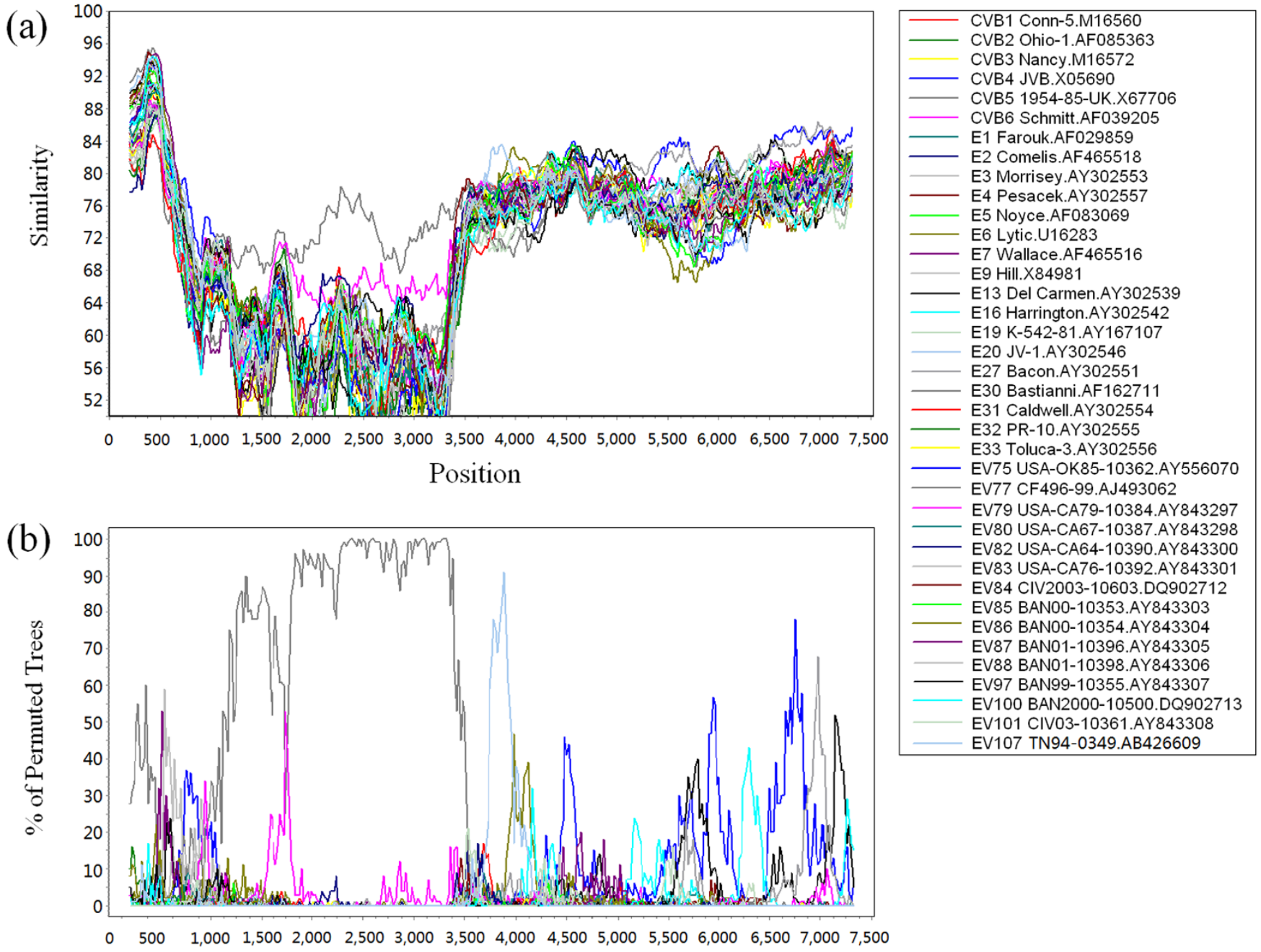

\section{Position}

Figure 3 Similarity plot (a) and bootscanning analysis (b) of Yunnan EV-B106 strain with EV-B prototype strains. The analysis was conducted via Simplot v3.5.1 using a sliding window of 400 nucleotides moving in steps of 20 nucleotides. The genome of strain 148/YN/CHN/12 serves as a query sequence. One possible recombination event between 12148/YN and EV-B107 is suggested in the 2B region (about nt positions 3700-3960).

Netherlands ${ }^{25}$; the E25 strain HN-2 (HM031191) from Henan Province, China; and the E30 strain 2012EM161 (KC897073) from Guangdong Province, China. Because the whole genome sequence of the prototype strain of EV-B106 cannot be obtained, the prototype strain of EV-B77 (the closest type in the VP1 region) was included in the analysis. The similarity plot analysis revealed multiple recombination events in the genome sequence of strain 12148/YN, and bootscanning analysis confirmed these recombination events (Fig. 4). In the $P 1$ coding region, $12148 / \mathrm{YN}$ had the highest similarity with the EV-B77 prototype strain. In the 5' UTR region, it had high similarity with E9 strain DM33 and E4 strain AUS250G. In the $P 2$ region, it had the highest similarity with E6 strain KM57/09, followed by E30 strain 2012EM161. In nt positions 4,940-7,280 (the partial $P 2$ and entire $P 3$ coding region), it had the highest similarity with E25 strain HN-2 (HM031191).

\section{Discussion}

There is no specific EV surveillance system in place for the mainland of China, although AFP surveillance, established as part of the Global Polio Eradication Initiative, has been conducted since 1994. As non-polio enteroviruses (NPEVs) can be isolated via the AFP surveillance, investigation of these viruses can provide valuable information on the molecular epidemiology of local EVs. Since the introduction of the molecular typing method, a considerable number of new EV types have been identified ${ }^{26,27}$. In addition, many newer EV types have been reported from retrospective investigations of the NPEV s from AFP surveillance conducted in the provinces of Yunnan and Shandong ${ }^{21,28,29}$. Here, for the first time, we report the whole genome sequence of a recently identified new type, EV-B106, which was also obtained from AFP surveillance.

Although the EV-B106 strain was isolated from stool samples of a patient with AFP during the course of onset, we could not collect supplementary data to conclude that EV-B106 was the causative agent in this case, because the virus was not isolated from the sites of pathology in the patient. Further surveillance data, such as seroepidemiology, on this virus in populations in the future might provide valuable information.

Yunnan is a frontier province in China and borders three countries: Vietnam, Laos, and Myanmar. The EV-B106 virus in this study 


\section{Nucleotide Position}

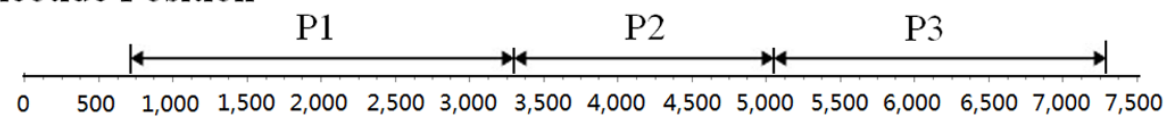

(a)
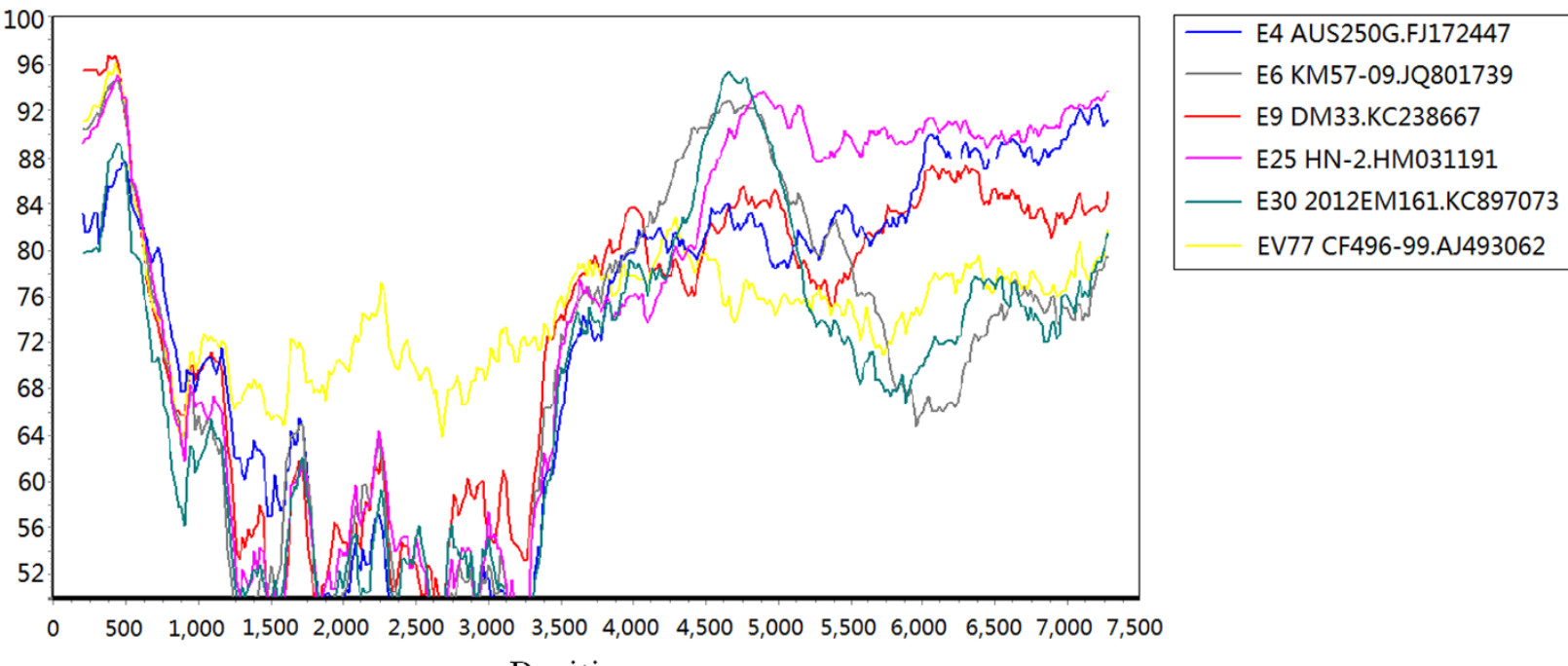

Position

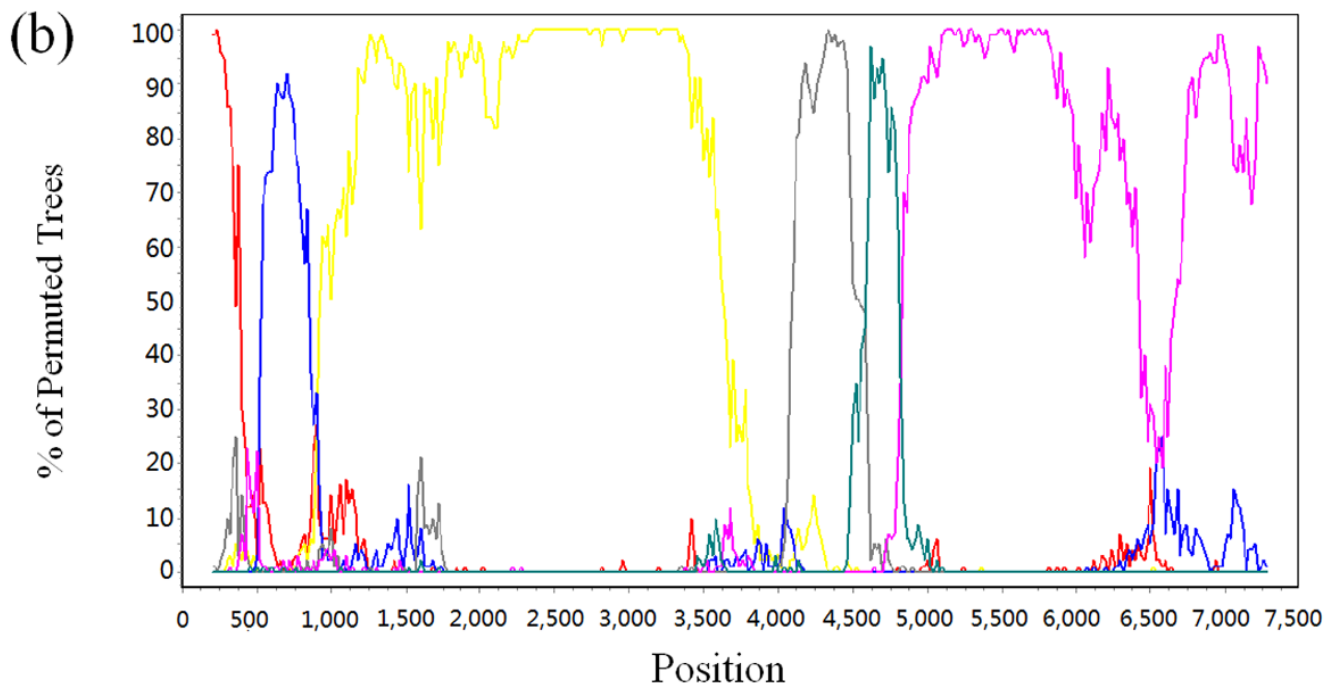

Figure $4 \mid$ Similarity plot (a) and bootscanning analysis (b) of the Yunnan EV-B106 strain with closely related strains. The analysis was conducted via Simplot v3.5.1 using a sliding window of 400 nucleotides moving in steps of 20 nucleotides. The genome of strain 148/YN/CHN/12 serves as a query sequence. Several possible recombination events are suggested in P2 and P3 coding regions.

was isolated in Honghe Prefecture, which is a region that borders Vietnam. For some villages near the border, daily communications across the border are very frequent, and surveillance for importation of medical pathogens should be of great concern.

EVs evolve rapidly (around 1-2\% per nt site per year), with most of the diversity in the form of nucleotide substitutions, deletions, or insertions $s^{30}$. Frame shifts in the VP3 coding region were found between the Yunnan EV-B106 strain (12148/YN) and the Pakistan EV-B106 strain. Taking the high divergence between the two EVB106 strains (81.3\% nt similarity in VP1 region) into consideration, the divergence and frame shifts between them indicate that the type has been circulating independently for a long time. Because most EV infections are subclinical, it is difficult to isolate the viruses in the initial stage of transmission.

Frame shifts were also found between the EV-B106 and EV-B77 strains. The VP1 nt similarity between EV-B77 and EV-B106 is
$73.3 \%$, which is not sufficient to distinguish strain $12148 / \mathrm{YN}$ from the existing types. However, two major frame shifts observed in the VP1 region result in 20.5\% (Yunnan strain) and 16.5\% (Pakistan strain) aa divergence from the EV-B77 prototype strain, which is great enough to separate the Yunnan strain from the EV-B77 strains $^{14}$, and classify it as a new type, EV-B106. EV-B77 has been reported to be isolated from children with gastroenteritis or AFP in USA, India, Kosovo, and Central African Republic, respectively ${ }^{1931-34}$. The complete VP1 nucleotide sequences of EV-B77 were found to diverge by $>29 \%$ from all prototypes ${ }^{19}$. However, the sequence data on EV-B77 was also in scarce in GenBank database, more genomic sequences are needed to further clarify the genetic relationship between EV-B77 and EV-B106.

In the $P 1$ region, continuous accumulation of nt substitutions and natural selection are well-known mechanisms of genome evolution ${ }^{35}$. The VP1 sequence is correlated with the types and can be used for 
classification of EVs. Conversely, in the P2 and P3 non-capsid regions, nt and aa sequences are relatively conserved within an EV species $^{36,37}$, and intra- and inter-serotypic recombination have usually been observed in these regions ${ }^{38-40}$. In our study, the Yunnan EVB106 strain is no exception_evidence of recombination with other EV-B strains in the $P 2$ and $P 3$ regions was found. Although similarity plot and bootscanning analysis suggested a recombination event between the Yunnan EV-B106 strain and EV-B107 prototype strains in the non-structural region, the percent support values were not high $(<85 \%)$. Since the recombination frequency of EV is very high and many prototype strains have been isolated several decades ago, it is often difficult (or impossible) to detect exact recombination partners if currently circulating strains are compared to the prototype strains. Therefore, the recombination analysis between EV-B106 strain 12148/YN and more closely related isolates were performed. In another approach, we screened some strains sharing high similarity with Yunnan EV-B106 in the 5' UTR, $P 2$, and $P 3$ regions by using BLAST, and performed a similar analysis (Fig. 4). Recombination events were observed between strain $12148 / \mathrm{YN}$ and the selected strains in the non-structural coding region with relatively higher percent support value ( $>90 \%)$. These selected strains were isolated from Australia, Finland, the Guangdong Province, and the Henan Province in China, and these regions are all geographically remote from the Yunnan Province. Hence, it is reasonable to conclude that long-term transmission of these viruses has taken place, so as to provide the spatial and temporal circumstances for recombination to occur.

In conclusion, this is the first report on the whole genome sequence of a recently described EV type, EV-B106. The extremely rare isolation rate suggests that it has not been a prevalent type in China, or even in the world, and the great genetic divergence among EV-B106 strains indicates that this type is not a newly emergent virus, but has circulated in the world for many years.

\section{Methods}

Virus isolation. The EV-B106 strain 12148/YN was isolated from an 18-month-old boy in the Honghe Prefecture of the Yunnan Province of China in July 2012. The patient initially had upper respiratory tract infection, and then developed asymmetric paralysis in his lower limbs. No residual paralysis was observed at the 60-day followup.

Stool samples from the child were collected and processed according to standard procedures recommended by the World Health Organization (WHO) ${ }^{41}$. Human rhabdomyosarcoma (RD) and human laryngeal epidermoid carcinoma (HEp-2) cell lines were used for virus isolation. Both cell lines were gifts from the WHO Global Poliovirus Specialized Laboratory in the USA and were all originally purchased from the American Type Culture Collection. A total of $200 \mu \mathrm{l}$ of chloroform-treated stool solution was added to each of the cell culture tubes. To ensure no cross contamination had occurred, cell tubes of normal RD and HEp- 2 cells served as negative controls. Infected cell cultures were harvested and used for further examination after a complete cytopathic effect had been obtained.

Amplification and sequencing. The viral RNA was extracted from the infected cell culture using a QIAamp Viral RNA Mini Kit (Qiagen, USA). Primer pairs 008-013 and 187-011, previously described by Oberste et al., were used for amplifying the entire VP1 coding region ${ }^{12,13}$. The reverse transcription-polymerase chain reaction (RT-PCR) was performed with the Access One-step RT-PCR Kit (Promega, USA) according to the manufacturer's instructions. Amplicons were purified and then sequenced using a BigDye Terminator v3.0 Cycle Sequencing Kit, and were analyzed using an ABI 3130 Genetic Analyzer (Applied Biosystems). The VP1 sequences obtained were compared with sequences available in the GenBank database by using the Basic Local Alignment Search Tool (BLAST) from the National Center for Biotechnology Information. Virus isolates showing $>75 \%$ nucleotide sequence identity with a known EV type were designated as the relative type ${ }^{13}$. The RT-PCR amplification on the rest of the genome was performed using six pairs of primers. Positive products were purified and sequenced as described above.

Phylogenetic and recombination analysis. Alignment of the whole genome sequences of strain $12148 / \mathrm{YN}$ and other EV types was performed using the BioEdit software (version 7.0.5.3) ${ }^{42}$. The phylogenetic trees were constructed with MEGA $4.0^{43}$ using the neighbor-joining method with a Kimura two-parameter model. Bootstrapping was performed with 1000 duplicates and bootstrap values greater than $80 \%$ were considered statistically significant for grouping. The SimPlot 3.5.1 program was used for similarity plot and bootscanning analysis ${ }^{44,45}$, with a $400 \mathrm{nt}$ window moving in $20 \mathrm{nt}$ steps and a Jukes-Cantor correction.
Nucleotide sequence accession number. The complete genome sequence of the EVB106 strain 12148/YN described in this study was deposited in the GenBank database under the accession number KF990476.

1. Khetsuriani, N., Lamonte-Fowlkes, A., Oberst, S. \& Pallansch, M. A. Centers for Disease Control and Prevention. Enterovirus surveillance--United States, 19702005. MMWR Surveill. Summ. 55, 1-20 (2006).

2. Faustini, A. et al. An outbreak of aseptic meningitis due to echovirus 30 associated with attending school and swimming in pools. Int. J. Infect. Dis. 10, 291-297 (2006).

3. Starlin, R. et al. Acute flaccid paralysis syndrome associated with echovirus 19 , managed with pleconaril and intravenous immunoglobulin. Clin. Infect. Dis. 33, 730-732 (2001).

4. Zhu, Z. et al. Molecular epidemiological analysis of echovirus 19 isolated from an outbreak associated with hand, foot, and mouth disease (HFMD) in Shandong Province of China. Biomed. Environ. Sci. 20, 321-328 (2007).

5. Pelletier, J. \& Sonenberg, N. Internal initiation of translation of eukaryotic mRNA directed by a sequence derived from poliovirus RNA. Nature. 334, 320-325 (1988).

6. Molla, A., Jang, S. K., Paul, A. V., Reuer, Q. \& Wimmer, E. Cardioviral internal ribosomal entry site is functional in a genetically engineered dicistronic poliovirus. Nature. 356, 255-257 (1992).

7. Chen, C. Y. \& Sarnow, P. Initiation of protein synthesis by the eukaryotic translational apparatus on circular RNAs. Science 268, 415-417 (1995).

8. Pilipenko, E. V., Maslova, S. V., Sinyakov, A. N. \& Agol, V. I. Towards identification of cis-acting elements involved in the replication of enterovirus and rhinovirus RNAs: a proposal for the existence of tRNA-like terminal structures. Nucleic. Acids. Res. 20, 1739-1745 (1992).

9. Pilipenko, E. V. et al. Cis-element, oriR, involved in the initiation of $(-)$ strand poliovirus RNA: a quasi-globular multi-domain RNA structure maintained by tertiary ('kissing') interactions. EMBO J. 15, 5428-5436 (1996).

10. Mirmomeni, M. H., Hughes, P. J. \& Stanway, G. An RNA tertiary structure in the 39 untranslated region of enteroviruses is necessary for efficient replication. J. Virol. 71, 2363-2370 (1997).

11. Enteroviruses: Polioviruses, coxsackieviruses, echoviruses, and newer enteroviruses. Fields Virology, 5th ed. (Lippincott Williams \& Wilkins, Philadelphia, 2007).

12. Oberste, M. S. et al. Comparison of classic and molecular approaches for the identification of untypeable enteroviruses. J. Clin. Microbiol. 38, 1170-1174 (2000).

13. Oberste, M. S., Maher, K., Kilptrick, D. R. \& Pallansch, M. A. Molecular evolution of the human enteroviruses: correlation of serotype with VP1 sequence and application to picornavirus classification. J. Virol. 73, 1941-1948 (1999).

14. Brown, B. A. et al. Resolving ambiguities in genetic typing of human enterovirus species C clinical isolates and identification of enterovirus 96,99 and 102. J. Gen. Virol. 90, 1713-1723 (2009).

15. Picornaviridae. Virus Taxonomy: Classification and Nomenclature of Viruses: Ninth Report of the International Committee on Taxonomy of Viruses. (Elsevier, San Diego, 2011).

16. Oberste, M. S. et al. Molecular identification of 13 new enterovirus types, EV7988, EV97, and EV100-101, members of the species Human Eneterovirus B. Virus Res. 128, 34-42 (2007).

17. Oberste, M. S. et al. Molecular identification and characterization of two proposed new enterovirus serotypes, EV74 and EV75. J. Gen. Virol. 85, 3205-3212 (2004).

18. Oberste, M. S. et al. Enteroviruses 76, 89, 90 and 91 represent a novel group within the species Human enterovirus A. J. Gen. Virol. 86, 445-451 (2005).

19. Norder, H. et al. Sequencing of 'untypable' enteroviruses reveals two new types, EV-77 and EV-78, within human enterovirus type B and substitutions in the BC loop of the VP1 protein for known types. J. Gen. Virol. 84, 827-836 (2003).

20. Shaukat, S. et al. Characterization of a novel enterovirus serotype and an enterovirus EV-B93 isolated from acute flaccid paralysis patients. PLoS ONE 8, e80040 (2013).

21. Bingjun, T. et al. Molecular typing and epidemiology of non-polio enteroviruses isolated from Yunnan province, the People's Republic of China. J. Med. Virol. 80, 670-679 (2008).

22. Andersson, P., Edman, K. \& Lindberg, A. M. Molecular analysis of the echovirus 18 prototype: evidence of interserotypic recombination with echovirus 9 . Virus Res. 85, 71-83 (2002).

23. Huang, T. et al. Evidence of recombination and genetic diversity in human rhinoviruses in children with acute respiratory infection. PLOS ONE 4, e6355 (2009).

24. Markey, P. G., Davis, J. S., Harnett, G. B., Williams, S. H. \& Speers, D. J. Meningitis and a febrile vomiting illness caused by echovirus type 4, Northern Territory, Australia. Emerg. Infect. Dis. 16, 63-68 (2010).

25. Paananen, A. et al. A single amino acid substitution in viral VP1 protein alters the lytic potential of clone-derived variants of echovirus $9 \mathrm{DM}$ strain in human pancreaticislets. J. Med. Virol. 85, 1267-1273 (2013).

26. Sun, Q. et al. Transmission of human enterovirus 85 recombinants containing new unknown serotype HEV-B donor sequences in Xinjiang Uighur autonomous region, China. PLoS ONE 8, e55480 (2013). 
27. Wang, J. et al. Isolation and characterization of a Chinese strain of human enterovirus 74 from a healthy child in the Tibet Autonomous Region of China. Arch. Virol. 157, 1593-1598 (2012).

28. Tao, Z. et al. Isolation and genomic characterization of three enterovirus 90 strains in Shandong, China. Arch. Virol. 158, 479-483 (2013).

29. Tao, Z. et al. Complete genome sequence of enterovirus 87 isolated from a child with acute flaccid paralysis in China in 2000. Virus Genes 47, 156-159 (2013).

30. Oberste, M. S., Maher, K., Patterson, M. A. \& Pallansch, M. A. The complete genome sequence for an American isolate of enterovirus 77. Arch. Virol. 152, 1587-1591 (2007).

31. Bailly, J. L., Cardoso, M. C., Labbé, A. \& Peigue-Lafeuille, H. Isolation and identification of an enterovirus 77 recovered from a refugee child from Kosovo, and characterization of the complete virus genome. Virus Res. 99, 147-155 (2004).

32. Rao, D. C. et al. Non-polio enteroviruses and their association with acute diarrhea in children in India. Infect. Genet. Evol. 17, 153-161 (2013).

33. Bessaud, M. et al. Molecular characterization of human enteroviruses in the Central African Republic: uncovering wide diversity and identification of a new human enterovirus A71 genogroup. J. Clin. Microbiol. 50, 1650-1658 (2012).

34. Laxmivandana, R., Yergolkar, P., Gopalkrishna, V. \& Chitambar, S. D. Characterization of the non-polio enterovirus infections associated with acute flaccid paralysis in South-Western India. PLoS ONE 8, e61650 (2013).

35. Oberste, M. S., Maher, K. \& Pallansch, M. A. Evidence of frequent recombination within species human enterovirus B based on complete genomic sequences of all thirty-seven serotypes. J. Virol. 78, 855-867 (2004).

36. Brown, B., Oberste, M. S., Maher, K. \& Pallansch, M. A. Complete genomic sequencing shows that polioviruses and members of human enterovirus species $\mathrm{C}$ are closely related in the noncapsid coding region. J. Virol. 77, 8973-8984 (2003).

37. Oberste, M. S., Penaranda, S., Maher, K. \& Pallansch, M. A. Complete genome sequences of all members of the species Human enterovirus A. J. Gen. Virol. 85 1597-1607 (2004).

38. Lukashev et al. Recombination in circulating enteroviruses. J. Virol. 77, 10423-10431 (2003).

39. Oprisan, G. et al. Natural genetic recombination between co-circulating heterotypic enteroviruses. J. Gen. Virol. 83, 2193-2200 (2002)

40. Santti, J., Hyypiä, T., Kinnunen, L. \& Salminen, M. Evidence of recombination among enteroviruses. J. Virol. 73, 8741-8749 (1999).

41. Isolation and identification of polioviruses. WHO Polio laboratory manual, 4 th edn. (World Health Organization, Geneva, 2004).
42. Hall, T. A. BioEdit: a user-friendly biological sequence alignment editor and analysis program for Windows 95/98/NT. Nucl. Acids. Symp. 41, 95-98 (1999).

43. Tamura, K., Dudley, J., Nei, M. \& Kumar, S. MEGA4: molecular evolutionary genetics analysis (MEGA) software version 4.0. Mol. Biol. Evol. 24, 1596-1599 (2007).

44. Lole, K. S. et al. Full-length human immunodeficiency virus type 1 genomes from subtype C-infected seroconverters in India, with evidence of intersubtype recombination. J. Virol. 73, 152-160 (1999).

45. Salminen, M. O., Carr, J. K., Burke, D. S. \& McCutchan, F. E. Identification of breakpoints in Intergenotypic recombinants of HIV type 1 by bootscanning. AIDS Res. Hum. Retroviruses 11, 1423-1425 (1995).

\section{Acknowledgments}

This study was supported by the National Natural Science Foundation of China (project no. 81160198) (http://www.nsfc.gov.cn)

\section{Author contributions}

J.T., Z.T., Z.D. and W.X. conceived the study and drafted the paper, J.T., Z.T. and Y.Z. gathered and analyzed the data, and J.Z., B.T., Z.Z. and L.Z. helped to interpret results and contributed to the writing.

\section{Additional information}

Supplementary information accompanies this paper at http://www.nature.com/ scientificreports

Competing financial interests: The authors declare no competing financial interests.

How to cite this article: Tang, J.J. et al. Complete Genome Characterization of a Novel Enterovirus Type EV-B106 Isolated in China, 2012. Sci. Rep. 4, 4255; DOI:10.1038/ srep04255 (2014).

(i) $\Theta$ This work is licensed under a Creative Commons Attribution-

NonCommercial-NoDerivs 3.0 Unported license. To view a copy of this license, visit http://creativecommons.org/licenses/by-nc-nd/3.0 